\title{
Magnocellular training improves visual word recognition
}

\author{
Tara Chouake ${ }^{1,2}$, Tamar Levy ${ }^{3}$, Daniel C. Javitt ${ }^{2,4}$ and Michal Lavidor ${ }^{1,5 *}$ \\ ${ }^{1}$ Department of Psychology, Bar Ilan University, Ramat-Gan, Israel \\ ${ }^{2}$ Department of Psychiatry, Nathan Kline Institute for Psychiatric Research, Orangeburg, NY, USA \\ ${ }^{3}$ The Gonda Brain Research Center, Bar Ilan University, Ramat-Gan, Israel \\ ${ }^{4}$ Department of Psychiatry, Division of Brain Stimulation, Columbia University, New York, NY, USA \\ ${ }^{5}$ Department of Psychology, University of Hull, Hull, UK
}

\section{Edited by:}

Hubert R. Dinse, Ruhr-Universität

Bochum, Germany

Reviewed by:

Hidenao Fukuyama, Kyoto

University, Japan

Peter Sörös, University of Western

Ontario, Canada

*Correspondence

Michal Lavidor, Department of

Psychology, Bar llan University,

Ramat Gan 52900, Israel.

e-mail:michal.lavidor@gmail.com
Current research has shown that basic visual networks, such as the magnocellular system, may play a crucial role in reading deficits related to dyslexia. The current study explored the relationship between magnocellular activity and reading abilities; we examined the hypothesis that a repeated usage of the magnocellular stream may improve reading by strengthening crucial neural pathways. Visual training was conducted for five consecutive days using a motion detection task (magnocellular training) and a control task of pattern detection (parvocellular training). Reading abilities of skilled readers were measured before and after the training using a lexical decision task. It was found that low-grade visual training overall can improve speed of lexical decision, but there is some indication that magnocellular training may selectively relate to accuracy. This potential added benefit of accuracy is crucial, and indicates that magnocellular training may have an advantage to parvocellular or general visual training when it comes to reading. This result lends support to the role of basic visual systems in reading, and has potential implications for neurorehabilitation of reading-related deficits.

Keywords: reading, magnocellular pathway, parvocellular pathway, perceptual training

\section{INTRODUCTION}

The magnocellular visual network is a distinct perceptual pathway projecting from the lateral geniculate nucleus (LGN) to primary visual areas, and provides the majority of visual information that is extended dorsally toward the parietal cortex. This extended magnocellular-dominated dorsal stream is principally critical for detecting spatial relationships as well as rapid changes, which allows this network to be sensitive to motion (Ungerleider and Haxby, 1994). The magnocellular stream has also been suggested to be important for intact reading (Stein and Walsh, 1997). Correlations between fundamental magnocellular abilities, such as motion detection, and more complex visual tasks, such as reading (Conlon et al., 2004) indicate that shared neural structures or pathways may play a role in both movement processing and visual language processing. Indeed, magnocellular abilities have been positively correlated with word recognition performance; moreover, coherent motion detection as well as word recognition have both been shown to contain a right visual field advantage, underlining their connection to the "verbal" left hemisphere and indicating that magnocellular activity plays a role in left hemisphere language areas during visual language processing (Levy et al., 2010).

The importance of proper magnocellular function in reading has been further supported by research on dyslexia, a neurological syndrome characterized by a reading disability. A substantial minority of children have developmental dyslexia, a specific difficulty in acquiring literacy skills. Children with developmental dyslexia fail to read at a level that is consistent with their IQ, motivation, and level of education (Ferrer et al., 2010), and often experience a sensation that the letters are blurred or jumping around as they are trying to read (Stein and Walsh, 1997). Thus, dyslexia is traditionally defined as a discrepancy between reading ability and intelligence in children receiving adequate reading tuition. These difficulties occur in otherwise bright and able children who master other tasks well (Snowling, 2000). Currently, the most prevalent view regarding the cognitive basis of dyslexia is the phonological deficit theory claiming that the reading impairment is caused by a deficit in phoneme representation and manipulation, leading to impaired grapheme-to-phoneme conversion, with direct effects on reading aloud (Snowling, 2000). However when considering written text, although it is commonly understood that phonological deficits are the key to dyslexia, in some cases magnocellular deficits may account for reading impairment. Impairment in the magnocellular visual system has long been suggested and supported, and an approximate $75 \%$ of individuals with dyslexia have a visual processing deficit (Lovegrove et al., 1990). A particular kind of dyslexia, dysphoneidetic type, has been implicated in that statistic (Borsting et al., 1995; Ridder et al., 1997). Individuals with dyslexia have been repeatedly found to exhibit magnocellular-specific deficits, such as higher motion detection thresholds, than dyslexia-free controls (Cornelissen et al., 1995). Physiological evidence from electrophysiological recordings (Livingstone et al., 1991) and imaging techniques (Eden et al., 1996; Demb et al., 1998) supports these behavioral patterns of impaired response to magnocellular-biased stimuli. For example, Eden et al. (1996) demonstrated that, compared 
to controls, individuals with dyslexia exhibit reduced functional activation during a motion detection task in visual area MT/V5, a region dominated by magnocellular input.

Taken together, it is likely that magnocellular dysfunction is a central component in reading-related disabilities of dyslexia, and it is thus probable that remediation of these perceptual areas can play a key role in corrective treatments. However, critics of the magnocellular reading theory (Amitay et al., 2002; Ramus et al., 2003; Sperling et al., 2005) have suggested an epiphenomenal link between dyslexia and dorsal stream dysfunction rather than a causative link, and thus more causative and intervention-based research is necessary to solidify the distinct role of dorsal stream function in reading.

This study aims to support the unique contribution of magnocellular function to reading as well as to demonstrate the potential of sensory stimulation in improving reading. We aimed to determine whether improving magnocellular-dominated ability could improve reading ability. If visual magnocellular training can improve reading ability, it indicates the involvement of this system in the natural process of reading. We suggest that repeated use of the predominately magnocellular dorsal stream can strengthen reading abilities by strengthening neural systems related to both. The training-based approach of this study can lend unique support to the magnocellular theory of reading because of its interventional nature.

Previous clinical methods have indicated that visual perception training can be a promising area for reading remediation. For example, it has been demonstrated that visual remediation with colored filters can play a role in reparation of reading ability in dyslexia (Ray et al., 2005). Following the same reasoning, the present study utilizes a training task that is strongly magnocellularly-biased: motion detection. Motion detection tasks are beneficial in that they have been related to a specific, localized area connected to the magnocellular system, MT/V5 (Zeki et al., 1991; Watson et al., 1993; Tootell et al., 1995; Silvanto et al., 2005). Because motion performance and its related area, V5, have been previously shown to be deficient in dyslexia both behaviorally and in functional imaging, it is a prime task for research on reading. Motion detection based training can potentially be a very practical tool for the future of perceptual treatment in dyslexia because of its ability to be attributed to a tangible neural structure that may have an element of plasticity. Perhaps other methods such as brain stimulation share this property; however, training is the easiest, cheapest, and most risk-free method of all.

Our magnocellular-based intervention was repeated for five consecutive days and contained motion detection via a random dot kinetogram. A lexical decision task was completed before and after the repeated visual stimulation in order to assess word recognition. A control training group was administered a pattern detection task, which contained a pattern of parallel lines rather than moving dots. The control task was matched in presentation parameters to the motion detection task, but has been shown to be dependent upon different cortical structures (Silvanto et al., 2005). Thus, this task is a sound control because it rules out the possibility that improvement found in reading could be caused by general visual training effects.

\section{METHODS}

\section{PARTICIPANTS}

Thirty-five healthy adults ( 11 males; mean age $24 \pm 3$ years) with no history of neurological or psychiatric disorder took part in the visual training study. All participants were native Hebrew speakers with normal or corrected-to-normal vision and with right hand dominance, having an average handedness score of at least 95 as evaluated using the Edinburgh Handedness Inventory (Oldfield, 1971). Tasks were carried out with participants' understanding and written consent, under a protocol approved by the local ethics committee and in accord with The Code of Ethics of the World Medical Association (Declaration of Helsinki).

\section{BEHAVIORAL TASKS}

Behavioral tasks were built and run using E-prime 1.1 software (Psychology Software Tools, Inc., PA: USA). Tasks were carried out in a darkened room and displayed on an $18^{\prime \prime}$ CRT monitor (refresh rate: $60 \mathrm{~Hz}$ ) at a viewing distance of $57 \mathrm{~cm}$.

\section{Motion detection}

Following Silvanto et al. (2005), the motion stimulus consisted of 80 yellow dots (1 pixel each) presented on a black background. Dots were placed at random positions within an imaginary square subtending $1.45 \times 1.45^{\circ}$ of visual angle, and were either stationary or moved coherently rightwards or leftwards within this virtual square. The displacement of the dots was 1 pixel per frame. Each trial began with a $500 \mathrm{~ms}$ fixation period, followed by a blank screen for $400 \mathrm{~ms}$, after which the stimulus appeared. Task included two sets of 80 trials with "motion" and "no motion" trials appearing randomly in equal probabilities (40 trials each). The two sets of 80 trials were separated by a one minute break. Subjects were instructed to report whether or not they detected motion in the display (regardless of its direction) by pressing a keyboard button. The duration of stimulus presentation was three frames of $16 \mathrm{~ms}$ each (stimulus duration of $50 \mathrm{~ms}$ total) and the ratio of moving dots in the "motion" trials was 15 moving dots to 65 stationary dots.

A practice session proceeded each of the five sessions. Initially, stimuli were presented for $48 \mathrm{~ms}$ (three frames) and motion trials included 50\% moving dots (40/40). The practice task was then made more difficult by changing the threshold to $30 / 50$. The threshold was finally adjusted (15/65). Each participant was required to achieve a $d^{\prime}$ of at least 1.0 in this practice round in order to ensure that participants could detect motion at this threshold. One participant did not reach the required minimal $d^{\prime}$ in the practice rounds, and was excluded from further data evaluation.

\section{Parallel line detection}

Following Silvanto et al. (2005), a corresponding and comparable task to the motion detection task was used as a parvocellular task. Just as in the motion detection task, the parallel line detection task consisted of 80 yellow dots ( 1 pixel each) presented on a black background. The parallel line stimuli was composed of two vertical columns, each consisting of six dots ( 1 pixel each, extending $0.72^{\circ}$ of visual angle vertically, separated by a distance of 5 pixels). Sixty-eight noise dots were placed at random positions 
within the imaginary square subtending $1.45 \times 1.45^{\circ}$ of visual angle. Subjects were instructed to report whether or not they detected parallel lines in the display by pressing a keyboard button. The duration of stimulus presentation was identical to the motion detection task, as were other viewing conditions. A practice round was administered prior to each of the five sessions, and subjects were required to achieve a minimum $d^{\prime}$ of 1.0 in the practice round.

\section{Lexical decision}

Stimuli consisted of 75 Hebrew five-letter strings of three types: words (46\%), adjacent anagrams (27\%), and non-words (27\%) (e.g., first, frist, and renus, respectively). Two such lists were created to avoid repetition of stimuli between the sessions. Letter strings were presented at the center of the screen in black over a light gray background using Courier New font, size 13. At a viewing distance of $57 \mathrm{~cm}$, each letter subtended $0.55^{\circ}$ vertically.

Each trial began with a $1000 \mathrm{~ms}$ fixation period after which the target string was displayed for $64 \mathrm{~ms}$. Target presentation was followed by a $1700 \mathrm{~ms}$ blank screen allowing the subject's response. Participants were asked to state whether the string was a legal Hebrew word or not by pressing a keyboard button. Accuracy and reaction time (RT) data were collected automatically for each subject. Six practice trials were given at the beginning of the session in order to familiarize subjects with the task. Two distinct word sets were counterbalanced among participants, one administered in the first session (prior to the visual training) and the other administered in the final session (post visual training).

\section{PROCEDURE}

The motion detection task was administered to the magnocellular training group, and the parallel line detection task was administered to the parvocellular training group. In the training groups, the respective visual task was repeated for five consecutive days, with the first and last sessions at the same approximate time of day (within $3 \mathrm{~h}$ ). The lexical decision task was administered on the first day, prior to training, and on the last day, post-training. A no-training group was also conducted, in which the lexical decision task was administered on the first and last day without visual training sessions. Accuracy and RT were recorded for all tasks.

To ensure randomization, subject numbers were written down, and each subject number was randomly assigned to group (using RANDOM function in Excel). At the end of the process some re-assignments were conducted in order to equate number of subjects between the groups. When the actual experiment started, subjects were given the pre-prepared subjects numbers and group number based on initial grouping. Since the no-training group had a different schedule than the training groups, and the meetings were pre-arranged with the participants, assignment to this group could not be entirely randomized. However, participants in this group were still naïve to the setup of the experiment, the purpose of the experiment, and the fact that there were other groups.

\section{DATA SCREENING AND TREATMENT}

Three subjects were excluded from the final analysis. One individual did not meet the inclusion criteria for effective magnocellular training, as described above, and was thus excluded from the magnocellular group. One individual was found to have previously taken part in experimental tasks included in this experiment and was thus not naïve; the final individual was excluded due to extremely low accuracy values in the lexical decision task (below two standard deviations). In the final sample, there were 11 participants in the magnocellular group, 11 participants in the parvocellular group and 10 participants in the no-training group. The groups did not significantly differ on age, gender distribution, and education level (see Table 1). All correct RT data within two standard deviations of the mean were included in the analysis, ensuring a normal distribution.

Due to ceiling effects in the lexical decision task and in order to factor in false alarm rates, $d$-prime values were used to evaluate the accuracy of subjects' performance. Two $d$-prime variables were calculated using the formula $d$-prime $=z$ (hit) $-z$ (false alarm). The first used word as the target (hit) and both adjacent anagrams and non-words as the control (false alarm). This was calculated in order to determine whether there was improvement in the lexical decision task overall. This variable was termed $D$-prime Word. The next $d$-prime calculated adjacent anagrams as the target and used words as the control, to determine the ability to distinguish anagrams from words. This variable was termed $D$-prime Anagram.

\section{RESULTS}

\section{REACTION TIME}

A three-way repeated measures ANOVA was conducted, with word type (Word, Adjacent Anagram) and time (sessions one

Table 1 | Descriptive statistics of age, years of education, and gender for training groups.

\begin{tabular}{|c|c|c|c|c|c|}
\hline & \multirow[t]{2}{*}{ Magnocellular training } & \multirow[t]{2}{*}{ Parvocellular training } & \multirow[t]{2}{*}{ No training } & \multicolumn{2}{|c|}{ Between group comparison } \\
\hline & & & & Value & Significance \\
\hline Age & $\mu=23.9 \pm 2.4$ & $\mu=23.3 \pm 2.5$ & $\mu=25.9 \pm 3.3$ & $F=2.6$ & 0.094 \\
\hline Years of education & $\mu=13.0 \pm 1.6$ & $\mu=14.1 \pm 3.1$ & $\mu=15.0 \pm 2.7$ & $F=1.7$ & 0.21 \\
\hline Gender & $\begin{array}{l}n \text { (women) }=7 \\
n(\text { men })=4\end{array}$ & $\begin{array}{l}n(\text { women })=8 \\
n(\text { men })=3\end{array}$ & $\begin{array}{l}n(\text { women })=8 \\
n(\text { men })=2\end{array}$ & $X^{2}=0.70$ & 0.70 \\
\hline
\end{tabular}

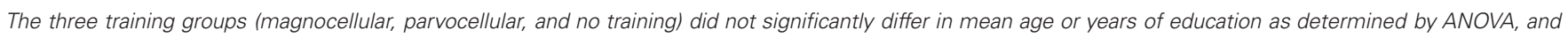
did not differ in gender distribution as determined by a chi-squared test. 
and two) as within subject factors, and group (Magnocellular training, Parvocellular training, No training) as between subject factors. There was a significant main effect for word type, with word recognition at a faster RT (mean $=647.6, \mathrm{SD}=15.6)$ than adjacent anagrams (mean $=785.2, \mathrm{SD}=24.7)$. There was also a main effect for time, where RT at session two (mean $=698.6$, $\mathrm{SD}=19.7)$ was quicker overall than at session one $($ mean $=734.2$, $\mathrm{SD}=20.3)$.

There was a significant interaction between time and group $(F(2,29)=3.98, p=0.03)$, with the two training groups decreasing in RT between sessions, and the no-training group remaining statistically unchanged.

Crucially, there was a significant three-way interaction between time, group, and word type $(F(2,29)=3.50, p=0.043)$ wherein both training groups improved in speed between the two sessions when responding to adjacent anagrams, while the group without training retained the same performance level in between the two sessions (see Table 2).

\section{ACCURACY}

A three-way repeated measures ANOVA was conducted, with word type (Word, Adjacent Anagram) and time (sessions one and two) as within subject factors, and group (Magnocellular training, Parvocellular training, No training) as between subject factors. In contrast to the RT results, no significant interaction between time and group was evident. Means and standard deviations for each group and session can be seen in Table 3 .

In order to assess more subtle connections between training procedures and accuracy of performance in the lexical decision task, correlations were computed between improvement in training and final scores on the lexical decision task. An improvement index was computed for the difference in RT between the first and final sessions of the visual training. A significant Pearson correlation ( $r=0.576, p=0.032)$ was observed only for the magnocellular training group between the improvement index and the two $d$-prime variables (see Figure 1 and Table 4). The parvocellular training group did not show significant correlations between improvement in training and $d$-prime scores at the post-training session.

\section{DISCUSSION}

The current study tested the potential of repeated sensory stimulation to improve reading and the contribution of magnocellular function to that process. The results indicate that low visual training, whether motion based or form based, can assist in letter string recognition, as evidenced by the significant improvement on the lexical decision task for both training groups as compared to the group without visual training.

Interestingly, this post-training improvement in RT was found specifically when participants were presented with adjacent anagrams. This exclusive improvement in anagram recognition following visual training could be due to the inherent difficulty in identifying this string type, which looks very similar to real words (e.g., frist vs. first), especially when it is displayed at high-speed. This difficulty is evident in high initial RTs, leaving greater room for improvement. However, the fact that such improvement did not manifest in the no-training group supports the contribution of rapid visual training to the process of anagram recognition.

While both types of visual training facilitated anagram recognition, there was a unique connection between the magnocellular training and the ability to improve in accuracy. It is important to note that the improvement in magnocellular training was associated with performance on both words and anagrams. Subject's sensitivity to words and anagrams following repeated

Table 2 | Mean reaction time $( \pm S D)$ on a lexical decision task by type, group, and session.

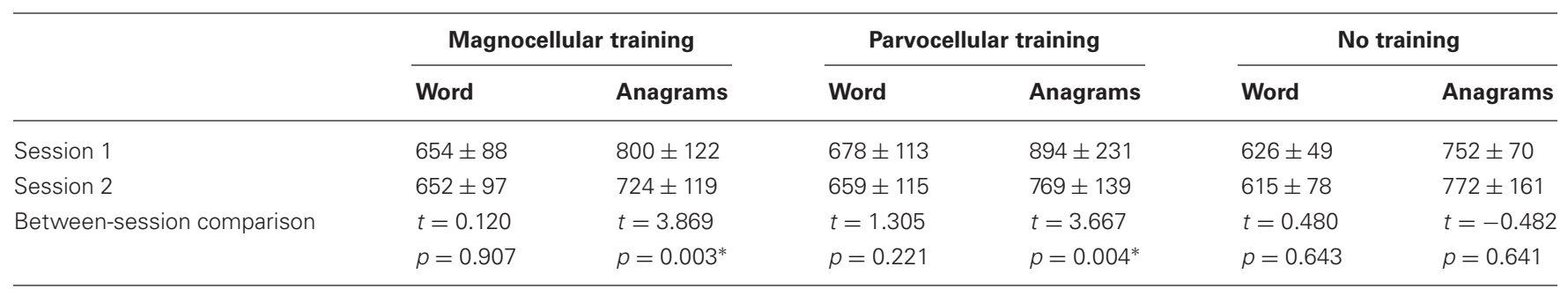

The mean reaction time $( \pm S D)$ are displayed for each training group and for each string type for sessions one and two (units of ms). The results of post-hoc, paired sample, two-tailed t-tests comparing performance in the two sessions are displayed in the last row. The two visual training groups significantly improved in recognizing adjacent anagrams; the no training group remained statistically equivalent between the two sessions. ( $\left.{ }^{*} p<0.05\right)$.

Table 3 | Mean accuracy ( $($ SD) on a lexical decision task by type, group, and session.

\begin{tabular}{|c|c|c|c|c|c|c|}
\hline & \multicolumn{2}{|c|}{ Magnocellular training } & \multicolumn{2}{|c|}{ Parvocellular training } & \multicolumn{2}{|c|}{ No training } \\
\hline Session 2 & $0.91 \pm 0.08$ & $0.92 \pm 0.15$ & $0.91 \pm 0.09$ & $0.86 \pm 0.1$ & $0.92 \pm 0.05$ & $0.83 \pm 0.23$ \\
\hline
\end{tabular}

The accuracy scores $( \pm S D)$ are displayed for each training group and for each string type for sessions one and two. 


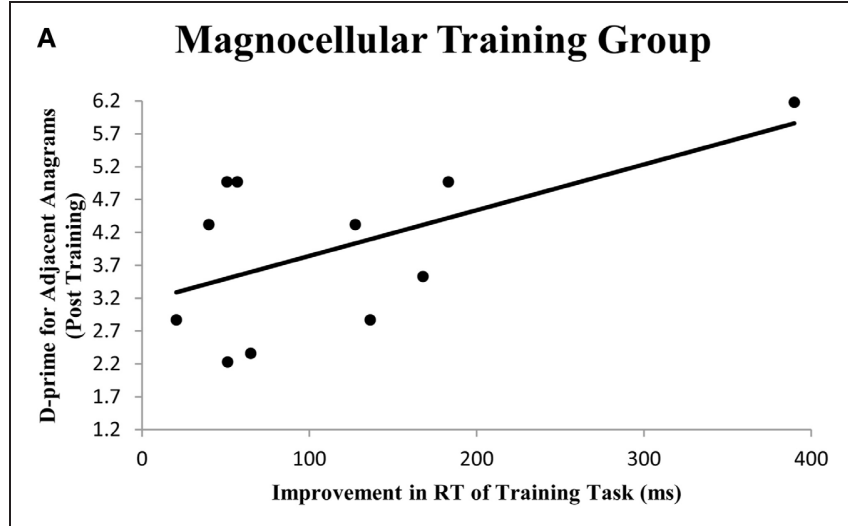

B Parvocellular Training Group

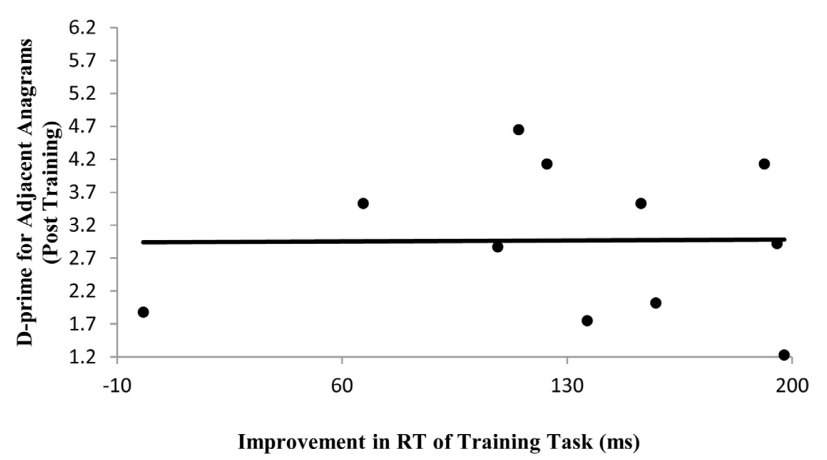

FIGURE 1 | Scatterplot between an improvement variable for performance on visual training and a $d$-prime variable for performance on a lexical decision task post-training. While RT improvement in magnocellular training $(\mathbf{A})$ correlated with the $d$-prime for adjacent anagrams ( $r=0.576, p=0.032$ ), improvement in parvocellular training

(B) did not ( $r=0.010, p=0.488)$. A similar pattern was found for words (see Table 3; graph not shown).

Table 4 | Pearson correlations between an improvement variable for performance on visual training and a $d$-prime variable for performance on a lexical decision task post-training.

\begin{tabular}{lll}
\hline & $\begin{array}{l}\text { Improvement index } \\
\text { magnocellular training }\end{array}$ & $\begin{array}{l}\text { Improvement index } \\
\text { parvocellular training }\end{array}$ \\
\hline D-prime Anagram & $r=0.576$ & $r=0.010$ \\
& $p=0.032^{*}$ & $p=0.488$ \\
D-prime Word & $r=0.540$ & $r=0.102$ \\
& $p=0.043^{*}$ & $p=0.383$ \\
\hline
\end{tabular}

D-prime Anagram refers to the post-training d-prime score for adjacent anagrams, and D-prime Word refers to the post-training d-prime score for real words. While improvement on magnocellular training significantly correlated with post-training $d$-prime scores on adjacent anagrams and real words, there was no significant correlation between improvement in parvocellular training and the lexical decision d-primes. (* $p<0.05)$.

magnocellular stimulation was correlated with their success in the training process. This added benefit of improving accuracy is crucial, and indicates that magnocellular training may have an advantage to general visual training when it comes to reading.
In light of our results, showing different effects for magnocellular and parvocellular based training, the role of the magnocellular system in reading can be interpreted by more than one model.

If we attribute the improvement in anagram recognition to a general visual training effect, then the magnocellular system appears to make a unique contribution to word recognition, above this overall effect. A specific connection to word recognition can be explained in terms of the integrated model of visual processing (Bullier, 2001). The M-system might enhance word recognition by a rapid delivery of a low-pass representation of the letter string, which is used to guide further processing in word-form related areas (Levy et al., 2010). Following that logic, magnocellular-based visual training is expected to improve the delivery of this low-pass representation thereby improving word recognition.

On the other hand, it might be the case that magnocellular training improved performance on both words and anagrams by improving the ability to discriminate between the two string types. Such an effect can be based on accurate letter position encoding. Cornelissen et al. (1998) suggested that the magnocellular system plays a role in accurate letter position encoding, possibly through precise shifting of visual attention during fixation (Vidyasagar, 1999). If this is the case, magnocellular-based visual training is expected to enhance correct letter ordering, thus improving the ability to detect real words as well as adjacent anagrams.

On the theoretical level, the question remains as to how the magnocellular system contributes to reading. However, on the practical level, our results demonstrate the potential of different types of visual training in improving higher visual processes such as word recognition. Moreover, magnocellular-guided training seems to have an advantage over general visual training in that it may operate on multiple string types and levels of performance (RT as well as accuracy).

These findings, though promising, should be interpreted with caution, since we did not measure neuronal changes following the training. The lack of observed neuronal changes cannot rule out the possibility that the observed improvement may contain various parts of neural and visual system as well as language reading neural substrates. Future studies might add brain imaging or recording to the training protocol to document the neural changes following specific protocols (see Schneiders et al., 2011). Additionally, increasing the training from low-grade to higher grade by lengthening duration of training can potentially enhance the trends seen in this experiment. Hopefully, this will also allow us to resolve the conflicting theoretical models. Lastly, low-grade training could be paired with neuroplastic promoting tools such as transcortical direct current stimulation (tDCS) over related cortical structures such as V5 in order to enhance the training effects.

\section{ACKNOWLEDGMENTS}

This study was supported by the Israel Academy of Sciences grant no. 100/10 and an ERC starting grant awarded to Michal Lavidor (Inspire 200512). The authors would like to thank the reviewers for their helpful comments, which have improved the manuscript. 


\section{REFERENCES}

Amitay, S., Ben-Yehudah, G., Banai, K., and Ahissar, M. (2002). Disabled readers suffer from visual and auditory impairments but not from a specific magnocellular deficit. Brain 125, 2272-2285.

Borsting, E., Ridder, W. H., Dudeck, K., Kelley, C., Matsui, L., and Motoyama, J. (1995). The presence of a magnocellular defect depends on type of dyslexia. Vision Res. 36, 1047-1053.

Bullier, J. (2001). Integrated model of visual processing. Brain Res. Brain Res. Rev. 36, 96-107.

Conlon, E., Sanders, M., and Zapart, S. (2004). Temporal processing in poor adult readers. Neuropsychologia 42, 142-157.

Cornelissen, P. L., Hansen, P. C., Gilchrist, I. D., Cormack, F., Essex, J., and Frankish, C. (1998). Coherent motion detection and letter position encoding. Vision Res. 38, 2181-2191.

Cornelissen, P., Richardson, A., Mason, A., Fowler, S., and Stein, J. (1995). Contrast sensitivity and coherent motion detection measured at photopic luminance levels in dyslexics and controls. Vision Res. 35, 1483-1494.

Demb, J. B., Boynton, G. M., and Heeger, D. J. (1998). Functional magnetic resonance imaging of early visual pathways in dyslexia. J. Neurosci. 18, 6939-6951.

Eden, G. F., Van Meter, J. W., Rumsey, J. M., Maisog, J. M., Woods, R. P., and Zeffiro, T. A. (1996). Abnormal processing of visual motion in dyslexia revealed by functional brain imaging. Nature 382, 66-69.

Ferrer, E., Shaywitz, A. B., Holahan, J. M., Marchione, K., and Shaywitz, S. E. (2010). Uncoupling of reading and IQ over time: empirical evidence for a definition of dyslexia. Psychol. Sci. 21, 93-101.

Levy, T., Walsh, V., and Lavidor, M. (2010). Dorsal stream modulation of visual word recognition in skilled readers. Vision Res. 50, 883-888.

Livingstone, M. S., Rosen, G. D., Drislane, F. W., and Galaburda, A. M. (1991). Physiological and anatomical evidence for a magnocellular defect in developmental dyslexia. Proc. Natl. Acad. Sci. U.S.A. 88, 7943-7947.

Lovegrove, W. J., Garzia, R. P., and Nicholson, S. B. (1990). Experimental evidence of a transient system deficit in specific reading disability. J. Am. Optom. Assoc. 61, 137-146.

Oldfield, R. C. (1971). The assessment and analysis of handedness: the Edinburgh inventory. Neuropsychologia 9, 97-113.

Ramus, F., Rosen, S., Dakin, S. C., Day, B. L., Castellote, J. M., White, S., and Frith, U. (2003). Theories of developmental dyslexia: insights from a multiple case study of dyslexic adults. Brain 126, 841-865.

Ray, N. J., Fowler, S., and Stein, J. F. (2005). Yellow filters can improve magnocellular function, motion sensitivity, convergence, accommodation, and reading. Ann. N.Y. Acad. Sci. 1039, 283-293.
Ridder, W. H., Borstin, E., Cooper, M., McNeel, B., and Huang, E. (1997). Not all dyslexics are created equal. Optom. Vis. Sci. 74, 99-104.

Schneiders, J. A. S., Opitz, B., Krick, C. M., and Mecklinger, A. (2011). Separating intra-modal and across-modal training effects in visual working memory: an fMRI investigation. Cereb. Cortex 21, 2555-2564.

Silvanto, J., Lavie, N., and Walsh, V. (2005). Double dissociation of V1 and V5/MT activity in visual awareness. Cereb. Cortex 15, 1736-1741.

Snowling, M. J. (2000). Dyslexia, 2nd edn. Oxford: Blackwell.

Sperling, A. J., Lu, Z. L., Manis, F. R., and Seidenberg, M. S. (2005). Deficits in perceptual noise exclusion in developmental dyslexia. Nat. Neurosci. 8, 862-863.

Stein, J., and Walsh, V. (1997). To see but not to read; the magnocellular theory of dyslexia. Trends Neurosci. 20, 147-152.

Tootell, R. B. H., Reppas, J. B., Kwong, K. K., Malach, R., Born, R. T., Brady, T. J., Rosen, B. R., and Belliveau, J. R. (1995). Functional analysis of human MT and related visual cortical areas using magnetic resonance imaging. J. Neurosci. 15, 3215-3230.

Ungerleider, L. G., and Haxby, J. V. (1994). 'What' and 'where' in the human brain. Curr. Opin. Neurobiol. 4, 157-165.

Vidyasagar, T. R. (1999). A neuronal model of attentional spotlight: parietal guiding the temporal. Brain Res. Brain Res. Rev. 30, 66-76.
Watson, J. D. G., Myers, R., Frackowiak, R. S. G., Hajnal, J. V., Woods, R. P., Mazziotta, J. C., Shipp, S., and Zeki, S. (1993). Area V5 of the human brain: evidence from a combined study using positron emission tomography and magnetic resonance imaging. Cereb. Cortex 3 , 79-94.

Zeki, S., Watson, J. D. G., Lueck, C. J., Friston, K. J., Kennard, C., and Frakowiak, R. S. J. (1991). A direct demonstration of functional specialization in human visual cortex. J. Neurosci. 11, 641-649.

Conflict of Interest Statement: The authors declare that the research was conducted in the absence of any commercial or financial relationships that could be construed as a potential conflict of interest.

Received: 30 September 2011; accepted: 24 January 2012; published online: 10 February 2012.

Citation: Chouake T, Levy T, Javitt DC and Lavidor M (2012) Magnocellular training improves visual word recognition. Front. Hum. Neurosci. 6:14. doi: 10.3389/fnhum.2012.00014

Copyright (C) 2012 Chouake, Levy, Javitt and Lavidor. This is an openaccess article distributed under the terms of the Creative Commons Attribution Non Commercial License, which permits non-commercial use, distribution, and reproduction in other forums, provided the original authors and source are credited. 